02

\title{
Низкочастотные молекулярные отклики в жидкости при регистрации сверхбыстрого оптического эффекта Керра
}

\author{
(C) В.Г. Никифоров \\ Казанский физико-технический институт им. Е.К. Завойского, \\ 420029 Казань, Россия \\ e-mail: vgnik@mail.ru
}

Поступила в редакцию 03.07.2018 г.

В окончательной редакции 03.07.2018 г.

Принята к публикации 20.11.2018 г.

Детально проанализированы низкочастотные молекулярные вращения в области $0-150 \mathrm{~cm}^{-1}$, которые регистрируются с высоким соотношением „сигнал/шум“ в сверхбыстром оптическом эффекте Керра (ОЭК), являющимся оптическим нелинейным откликом третьего порядка при нерезонансном возбуждении жидкости лазерными фемтосекундными импульсами. Показано, что редуцированная рамановская спектральная плотность (РСП), полученная из экспериментальных данных с помощью хорошо известной процедуры деконволюции, неоднозначна из-за проблемы разделения ориентационного и либрационного вкладов в совокупный сигнал ОЭК. Этот факт существенно ограничивает достоверность извлекаемой информации о межмолекулярных движениях в жидкости. На примере сверхбыстрого ОЭК в бензонитриле показано, что в диапазоне 0-300 fs вращательные отклики нельзя считать независимыми, и применение ряда дополнительных критериев, следующих из предположения об их корреляции, позволяет устранить указанную выше неопределенность в получении функции РСП из экспериментальных данных.

DOI: $10.21883 /$ OS.2019.03.47364.188-18

\section{Введение}

Традиционно молекулярная динамика находится в центре внимания, поскольку лежит в основе множества физических, химических и биологических процессов. Среди различных молекулярных типов движений слабо изученными остаются коллективные межмолекулярные движения в жидкости, которые включают в себя колебания, столкновения и либрации молекул с характерными частотами в терагерцовой области [1-3].

Фемтосекундная поляризационная спектроскопия с регистрацией сверхбыстрого оптического эффекта Керра (ОЭК) позволяет регистрировать времяразрешенные отклики молекулярных движений в жидкости с частотами от 0 до $600 \mathrm{~cm}^{-1}$, что открывает широкие возможности для детальных исследований [2,4-6]. Как показывает практика, частотно разрешенная спектроскопия (например, спектроскопия комбинационного рассеяния) из-за интенсивного релеевского крыла оказывается непригодной для регистрации сигнала в низкочастотной области $[2,7,8]$. Отметим также бурно развивающиеся методы терагерцовой спектроскопии [9], где анализ частотной зависимости диэлектрической проницаемости дает возможность изучать межмолекулярные колебаниях и структурные свойства конденсированных сред. Однако сложности генерации и регистрации терагерцового излучения ограничивают широкое применение этих методов. Таким образом, спектроскопия, основанная на регистрации ОЭК, в настоящее время является, пожалуй, наиболее доступным средством для исследований низкочастотных оптических откликов в конденсирован- ных средах с рекордно высоким соотношением „сигнал/шум“6.

В формирование сверхбыстрого ОЭК вносят вклады комбинационно активные колебательные и вращательные отклики. При этом возникает задача корректного разложения совокупного сигнала на составляющие компоненты. В настоящий момент предложен ряд способов анализа спектроскопической информации в сигнале ОЭК $[5,6,10]$. Однако следует признать, что отсутствие общепризнанного теоретического подхода для детального описания молекулярных движений в субпикосекундном диапазоне представляет существенное препятствие на пути решения этой задачи. В данной ситуации разумно ориентироваться на наиболее обоснованные методы интерпретации сигнала, позволяющие извлекать из экспериментальных данных информацию о спектральных характеристиках межмолекулярных движений. Именно таким методом является процедура деконволюции, предложенная в работах [11-13]. Она учитывает эффекты, связанные с конечной длительностью лазерных импульсов, и позволяет разделять сигнал на электронную и ядерную компоненты.

Изучение ядерной компоненты, которая содержит спектроскопическую информацию о низкочастотных движениях молекул в жидкости, как правило, основывается на анализе рамановской спектральной плотности $[5,6,13]$. Для получения спектра межмолекулярных движений, обусловленных особенностями локальной структуры жидкости, из рамановской спектральной плотности удаляют вклад, соответствующий внутримолекулярным колебаниям и ориентационным движениям 
молекул. Внутримолекулярные отклики имеют ярко выраженные интенсивные полосы с максимумами на частотах внутримолекулярных колебательных мод, что позволяет легко идентифицировать их в совокупном спектре. Однако корректное разделение вкладов когерентных ориентационных и межмолекулярных (либрационных) движений представляет собой сложную задачу.

Традиционный способ ее решения заключается в вычитании ориентационного вклада из сигнала сверхбыстрого ОЭК $[5,6,12,13]$. Ориентационная упорядоченность молекул, созданная интенсивным импульсом накачки, разрушается под действием процессов вращательной диффузии. Таким образом, предполагается, что ориентационный отклик $r_{\text {or }}(t)$ представляет собой экспоненциально затухающую функцию с характерными временами релаксации $\tau_{\text {or }}^{(i)}$ в субпикосекундном диапазоне:

$$
\begin{aligned}
r_{\mathrm{or}}(t) \sim & \left(A \exp \left(-\frac{t}{\tau_{\mathrm{or}}^{(1)}}\right)+(1-A) \exp \left(-\frac{t}{\tau_{\mathrm{or}}^{(2)}}\right)\right) \\
& \times\left(1-\exp \left(-\frac{t}{\gamma}\right)\right) .
\end{aligned}
$$

Параметр $\gamma$ симулирует скорость нарастания ориентационного отклика во время и после действия возбуждающего импульса, константа $A$ определяет относительный вклад слагаемых в выражении (1). Интуитивно значение $\gamma$ принимается равным $200 \mathrm{fs}$.

После вычитания ориентационного вклада из сигнала ОЭК (с симуляцией ориентационного отклика в виде (1)) и применения процедуры деконволюции получают редуцированную рамановскую спектральную плотность (РСП), которая отражает спектральные характеристики комбинационно-активных межмолекулярных движений, формирующих оптический нелинейный отклик третьего порядка. При этом вне поля зрения оказывается очень важный момент - зависимость РСП от выбора величины $\gamma$.

Именно этот аспект находится в центре внимания настоящей работы, поскольку в многочисленных исследованиях локальной структуры конденсированных сред вид РСП имеет ключевое значение [14-19]. Одним из способов решения этой проблемы может быть многоимпульсное возбуждение ОЭК, предложенное в работах [20-24]. Оно позволяет применять сценарии селективного возбуждения, где создаются условия для усиления одних и подавления других откликов комбинационно-активных молекулярных движений. В [24] был использован двухимпульсный сценарий для подавления ориентационной анизотропии в бензонитриле с целью выделения либрационного отклика в сигнале ОЭК. Недостатком этого метода являются технические трудности организации двухимпульсного возбуждения по сравнению с одноимпульсным, а также невозможность применения процедуры деконволюции.

В настоящей работе предлагается альтернативный подход для анализа вращательных откликов, в котором вводятся дополнительные предположения о корреляции между ориентационными и либрационными движениями молекул. Это дает возможность разделять вклады в сигнал ОЭК при традиционном одноимпульсном возбуждении, где удобно использовать формализм процедуры деконволюции. Приводятся аргументы, касающиеся адекватности выбора функции ориентационного отклика $r_{\text {or }}(t)$ в виде (1), рассматриваются условия выделения ориентационного вклада из сигнала ОЭК, а также указываются критерии выбора параметра $\gamma$. В качестве примера проводится анализ сигнала ОЭК с одноимпульсным возбуждением в бензонитриле (экспериментальные данные взяты из работы [24]). Также дано сравнение с результатами работы [24], где спектр молекулярных либраций получен из анализа экспериментальных данных по сверхбыстрому ОЭК в бензонитриле с двухимпульсным возбуждением.

\section{Оптический нелинейный отклик среды третьего порядка и редуцированная РСП}

Наблюдаемый в эксперименте сигнал сверхбыстрого ОЭК $S(\tau)$ описывается сверткой функции оптического нелинейного отклика среды $r(t)$ третьего порядка и автокорреляционной функции лазерных импульсов $G(t-\tau)[4-6]:$

$$
\begin{aligned}
S(\tau) \sim \int_{-\infty}^{\infty} r(t) G(t-\tau) d t, \\
G(x)=\int_{-\infty}^{\infty} I(x+t) I(t) d t,
\end{aligned}
$$

где $\tau$ - задержка между возбуждающим и пробным импульсами, $I(t)$ - огибающая интенсивности лазерного импульса. Функция отклика $r(t)$ является суммой независимых друг от друга „электронного“ $\sigma$ и „ядерного“ $d(t)$ молекулярных откликов:

$$
r(t)=\sigma+d(t)
$$

„Электронный“ отклик $\sigma$ отражает состояние поляризуемости молекул вследствие изменения конфигурации электронной плотности внешним полем, в результате чего вклад этого отклика в наблюдаемый сигнал повторяет форму автокорреляционной функции $G(\tau)$. Функция $d(t)$ представляет главный спектроскопический интерес, поскольку она связывает изменение поляризуемости среды с когерентными движениями молекулярных ядер.

Для разделения „электронного“ и „ядерного“ откликов применяется известная процедура деконволюции сигнала сверхбыстрого ОЭК [11-13], которая использует 
фурье-преобразование и свойства указанных молекулярных откликов:

$$
\begin{gathered}
d(t) \sim i H(t) \int_{-\infty}^{\infty} e^{-i \omega t} T(\omega) d \omega, \\
T(\omega)=\operatorname{Im}\left(\frac{\int_{-\infty}^{\infty} e^{i \omega t} S(t) d t}{\int_{-\infty}^{\infty} e^{i \omega t} G(t) d t}\right),
\end{gathered}
$$

где $H(t)$ - функция Хевисайда.

Как уже было отмечено выше, функция „ядерного“ отклика $d(t)$ представляет собой суперпозицию откликов разных молекулярных движений, которые по своему характеру можно разделить на внутримолекулярные колебания $r_{\mathrm{vib}}(t)$, ориентационную упорядоченность молекул в среде $r_{\text {or }}(t)$ и молекулярные либрации $r_{\text {lib }}(t)$, связанные с межмолекулярными взаимодействиями. Для изучения либрационного спектра из совокупного сигнала ОЭК $S(\tau)$ удаляется вклад ориентационного отклика $S_{\text {or }}(\tau)$ :

$$
\begin{gathered}
S_{R}(\tau)=S(\tau)-S_{\mathrm{or}}(\tau), \\
S_{\mathrm{or}}(\tau)=\int_{-\infty}^{\infty} r_{\mathrm{or}}(t) G(t-\tau) d t,
\end{gathered}
$$

где предполагается, что ориентационный отклик $r_{\text {or }}(t)$ имеет вид (1). Далее $S_{R}(\tau)$ подвергается процедуре деконволюции (6), в результате чего получают функцию РCП $T_{\mathrm{RSD}}(\omega)$.

В качестве примера рассмотрим зависимость формы $T_{\mathrm{RSD}}(\omega)$ от параметра $\gamma$ для сигнала бензонитрила $\mathrm{C}_{6} \mathrm{H}_{5} \mathrm{CN}$, экспериментальные данные которого представлены, например, в работах [24-28]. Рисунок 1 показывает сигнал ОЭК, полученный в работе [24], где огибающая интенсивности лазерных импульсов с несущей длиной волны $790 \mathrm{~nm}$ имела вид

$$
I(t)=\operatorname{sech}\left(-\frac{t}{\tau_{l}}\right)+0.17 \exp \left(-\frac{t^{2}}{\left(8 \tau_{l}\right)^{2}}\right)
$$

В (8) $\tau_{l}$ принимает значение $13 \mathrm{fs}$, соответствующее длительности импульса на полувысоте, равной $45 \mathrm{fs}$. Указанные параметры позволили эффективно возбуждать и регистрировать низкочастотные колебания в области $0-200 \mathrm{~cm}^{-1}$, куда попадает также внутримолекулярная колебательная мода $170 \mathrm{~cm}^{-1}$.

На рис. 2 представлена зависимость спектра $T_{\mathrm{RSD}}(\omega)$ от значений параметра $\gamma=0.01,0.2,0.6 \mathrm{ps}$. Хорошо видно, что значение $\gamma=0.01 \mathrm{ps}$ соответствует спектру $T_{\mathrm{RSD}}(\omega)$, который заметно отличается от остальных в области $100-160 \mathrm{~cm}^{-1}$. Особенно важно, что все спектры $T_{\mathrm{RSD}}(\omega)$ различаются в низкочастотной области $0-60 \mathrm{~cm}^{-1}$, связанной с молекулярными вращениями и либрациями. Необходимо заметить, что на основании описанной выше процедуры деконволюции не представляется возможным отдать предпочтение какому-либо варианту. Этот пример является яркой иллюстрацией

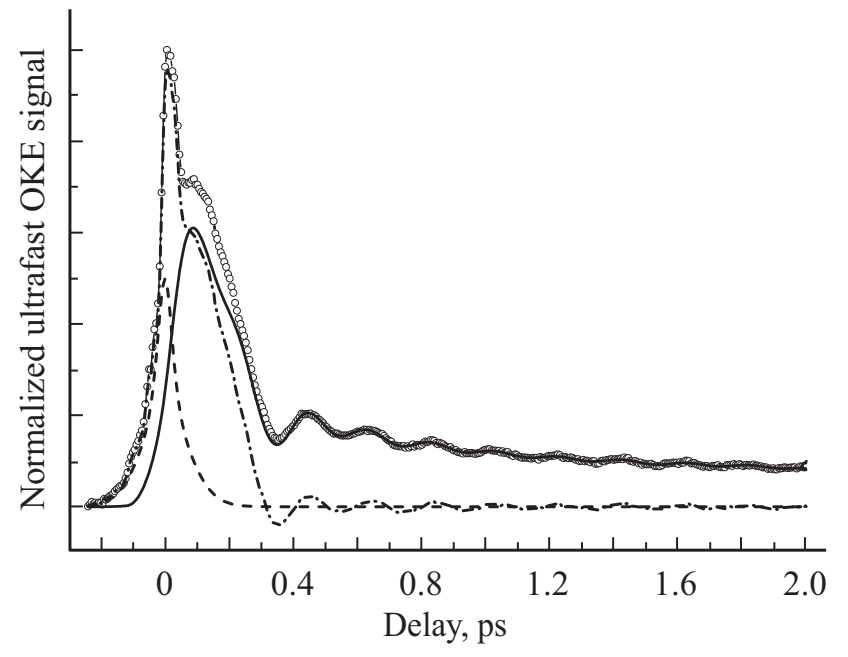

Рис. 1. Сигнал сверхбыстрого ОЭК в бензонитриле. Символами о обозначены экспериментальные данные, полученные в [24], сплошная линия - ядерный вклад $d(t)$ в сигнал ОЭК, штрихи - автокорреляционная функция лазерных импульсов $G(\tau)$, штрихпунктир - редуцированный сигнал ОЭК $S_{R}(\tau)$.

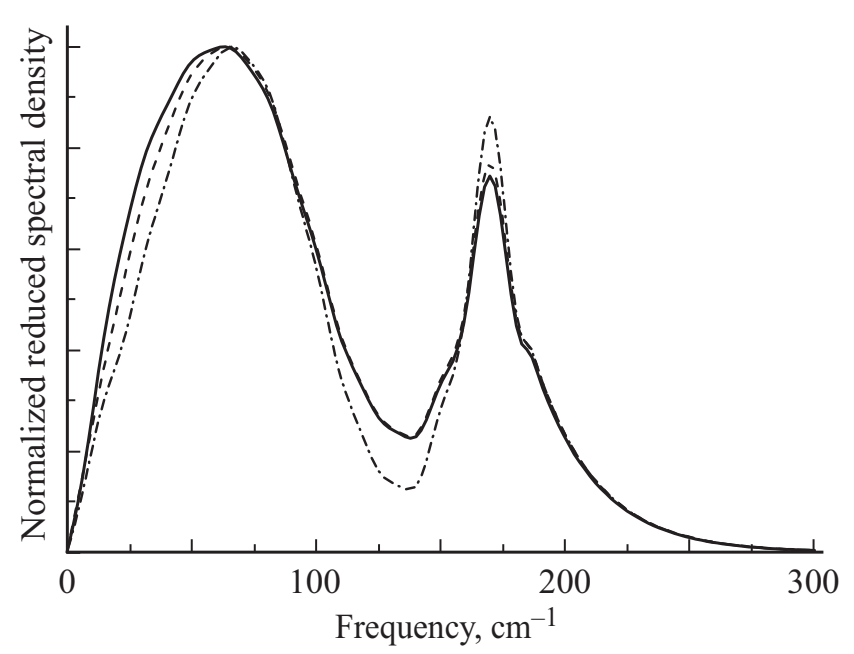

Рис. 2. Зависимость редуцированной рамановской спектральной плотности $T_{\mathrm{RSD}}(\omega)$ (вычисленной с помощью симуляции ориентационного отклика выражением (1) с параметрами $\tau_{\mathrm{or}}^{(1)}=0.4 \mathrm{ps}$ и $\tau_{\mathrm{or}}^{(2)}=8 \mathrm{ps}$ ) от значения $\gamma$ : сплошная линия $0.6 \mathrm{ps}$, штрихи $-0.2 \mathrm{ps}$, штрихпунктир $-0.01 \mathrm{ps}$.

проблемы разложения сигнала ОЭК на составляющие отклики, что в данном случае проявляется в неопределенности формы РСП, полученной при анализе экспериментального сигнала ОЭК в бензонитриле.

\section{Ориентационный отклик}

Согласно [10,29-32], ориентационная диффузия молекул обусловлена их вращательными прыжками в новые равновесные положения, и при малых углах прыжков 
такое движение описывается уравнением вращательной диффузии. Линейно поляризованный лазерный импульс с интенсивностью $I(t)$ стремится выстроить молекулы вдоль оси своей поляризации. Энергию взаимодействия импульса накачки с молекулой можно представить в виде

$$
W(\theta)=-\Delta \alpha \sin (\theta) I(t),
$$

где $\Delta \alpha-$ анизотропия поляризуемости молекулы, $\theta-$ угол между наведенным дипольным моментом молекулы и направлением поляризации лазерного импульса. Поток $j_{W}=\frac{d}{d t} \theta f(\theta, t)$ зависит от ориентационной функции распределения $f(\theta, t)$ молекул и интенсивности лазерного поля:

$$
\frac{d \theta}{d t}=-\frac{D}{k T} \nabla W(\theta),
$$

где $D-$ коэффициент диффузии, $k-$ постоянная Больцмана, $T$ - температура. С другой стороны, процессы вращательной диффузии препятствуют упорядочению молекул. Поток, обусловленный ими, пропорционален градиенту функции распределения $j_{D}=-D \nabla f(\theta, t)$. Если потребовать, чтобы полный поток удовлетворял уравнению непрерывности $\frac{\partial}{\partial t} f(\theta, t)=-\operatorname{div}(j)$, получим уравнение для функции распределения:

$$
\begin{aligned}
\frac{1}{D} \frac{\partial f(\theta, t)}{\partial t}= & \frac{1}{\sin (\theta)} \frac{\partial}{\partial \theta}\left\{\operatorname { s i n } \theta \left(\frac{\partial f(\theta, t)}{\partial \theta}\right.\right. \\
& +\Delta \alpha(I(t) / k T) \sin (2 \theta) f(\theta, t))\} .
\end{aligned}
$$

В условии слабого нерезонансного возбуждения при незначительном отклонении функции распределения от изотропного состояния выражение для ориентационной анизотропии $R_{\mathrm{or}}(t)$, вызванной действием лазерного импульса, принимает вид [10]

$$
R_{\mathrm{or}}(t) \propto \Delta \alpha^{2} \int_{0}^{\infty} I\left(t-t^{\prime}\right) \exp \left(-\frac{t^{\prime}}{\tau_{d}}\right) d t^{\prime}
$$

который связан соотношениями

$$
\begin{aligned}
R_{\text {or }}(t) & =\int_{0}^{\infty} I\left(t-t^{\prime}\right) r_{\text {or }}\left(t^{\prime}\right) d t^{\prime}, \\
S_{\text {or }}(\tau) & =\int_{0}^{\infty} I\left(t^{\prime}-\tau\right) R_{\text {or }}\left(t^{\prime}\right) d t^{\prime}
\end{aligned}
$$

с функцией ориентационного отклика $r_{\text {or }}(t)$ и ориентационной компонентой $S_{\text {or }}(\tau)$ сигнала сверхбыстрого ОЭК. Выражение (12) указывает, что ориентационный отклик имеет вид

$$
r_{\text {or }}(t) \propto H(t) \exp \left(-\frac{t}{\tau_{d}}\right) .
$$

Таким образом, затухающие экспоненциальные части в выражении (1) описывают релаксацию наведенной ориентационной анизотропии на основе механизмов вращательной диффузии. Недостатком выражения (13) является пренебрежение инерционными процессами, которые являются существенными при возникновении ориентационной анизотропии под действием лазерного импульса. Действительно, если предположить, что лазерный импульс имеет форму дельта-функции $I(t)=I_{0} \delta(t)$, то эволюция наведенной им анизотропии будет описываться функцией

$$
R_{\text {or }}(t) \propto H(t) I_{0} \exp \left(-t / \tau_{d}\right)
$$

которая подразумевает мгновенное появление анизотропии. Однако с физической точки зрения очевидно, что возникновение ориентационной анизотропии не может происходить быстрее молекулярных вращательных колебаний, т. е. либраций, которые имеют характерные частоты в диапазоне $0-100 \mathrm{~cm}^{-1}$. Таким образом, естественно ожидать, что нижний предел времени нарастания ориентационного отклика должен находиться в области $100 \mathrm{fs}$. Соответственно этому применение в эксперименте лазерных импульсов длительностью меньше $100 \mathrm{fs}$ создает условие для уверенной регистрации нарастания ориентационного вклада в сигнале ОЭК, которое не учитывается выражением (13). Именно этот факт является причиной появления множителя с параметром $\gamma$ в (1). При этом важно помнить, что выбранная форма множителя в виде $(1-\exp (-t / \gamma))$ отражает лишь необходимость симулировать плавное нарастание ориентационной анизотропии.

Наличие в (1) двух экспоненциально затухающих слагаемых $\mathbf{c}$ временами $\tau_{\mathrm{or}}^{(1)}$ и $\tau_{\mathrm{or}}^{(2)}$ является экспериментальным наблюдением для многих жидкостей $[5,6,25,27,28,33]$. Оно указывает, что в релаксации ориентационной анизотропии могут принимать участие два разных процесса вращательной диффузии. Например, это может быть обусловлено геометрией молекулы с разными моментами инерции относительно своих главных осей или локальным окружением, формирующим разные потенциальные барьеры относительно разных осей вращения. Могут быть и иные причины, связанные с неоднородностями локальной организации жидкости в фемтосекундном диапазоне. Таким образом, двух- (или более) экспоненциально затухающий ориентационный отклик можно интерпретировать как суперпозицию ориентационных откликов с разными релаксационными процессами. В этом случае корректно будет предположить, что каждому ориентационному процессу с временем релаксации $\tau_{\text {or }}^{(i)}$ соответствует собственный параметр $\gamma^{(i)}$, поэтому выражение для совокупного ориентационного отклика предпочтительнее искать в виде

$$
r_{\text {or }}(t) \sim \sum_{i=1}^{n} A_{i} \exp \left(-\frac{t}{\tau_{\text {or }}^{(i)}}\right)\left(1-\exp \left(-\frac{t}{\gamma^{(i)}}\right)\right)
$$

где $n$ - число релаксационных процессов ориентационной анизотропии, описываемых выражением (13). 


\section{Корреляция между либрационным и ориентационным откликами}

Представляется важным обсудить физические критерии времени нарастания ориентационного отклика, который симулируется параметром $\gamma$. Заметим, что в основе либрационного и ориентационного откликов лежат молекулярные вращения, поэтому считать эти отклики абсолютно независимыми друг от друга невозможно. Более того, одни и те же молекулярные вращения, инициированные лазерным импульсом, являются причиной возникновения обоих откликов. Когерентные либрации молекул приводят к неравновесному распределению молекул по либрационным углам, что в свою очередь регистрируется как либрационный отклик. Естественно принять, что вероятность вращательного перехода молекулы $v_{\text {or }}(\theta, t)$ в новое ориентационное положение растет с амплитудой либрационного угла $\theta_{\mathrm{lib}}(\theta, t)$, который характеризует отклонение молекул от текущего равновесного положения $\theta$ :

$$
v_{\text {or }}(\theta, t) \propto\left|\theta_{\text {lib }}(\theta, t)\right|
$$

В условиях эксперимента по регистрации сверхбыстрого ОЭК (т.е. незначительного отклонения функции распределения молекулярных ориентаций $f(\theta, t)$ от изотропного состояния), поток $j_{W}$, обусловленный взаимодействием молекул с полем возбуждающего импульса, значительно превышает поток диффузии $j_{D}$, вызванный наведенной ориентационной анизотропией среды, $j_{W} \gg j_{D}$. Таким образом, можно принять, что время нарастания ориентационного отклика $t_{\gamma}$ не зависит от процессов вращательной диффузии. Учитывая, что скорость нарастания ориентационного отклика пропорциональна вероятности перехода молекул в новые равновесные положения:

$$
\left|\frac{d r_{\text {or }}(t)}{d t}\right| \propto \int_{0}^{\pi} v_{\text {or }}(\theta, t) f(\theta, t) \sin (\theta) d \theta,
$$

а анизотропия среды, обусловленная молекулярными либрациями, пропорциональна величинам углов молекулярных либраций:

$$
R_{\mathrm{lib}}(t) \propto \int_{0}^{\pi} \theta_{\mathrm{lib}}(\theta, t) f(\theta, t) \sin (\theta) d \theta,
$$

с учетом выражения (15) получим

$$
\frac{d r_{\mathrm{or}}(t)}{d t} \propto R_{\mathrm{lib}}(t)
$$

Из (17) следует, что время нарастания ориентационного отклика $t_{\gamma}$ должно быть близко к длительности либрационного отклика: $t_{\gamma} \approx \tau_{\mathrm{lib}}$. Например, в случае равновесного распределения молекул по углам либрационный отклик отсутствует, и процессы вращательной диффузии приводят к релаксации ориентационного отклика, что как раз и наблюдается в эксперименте на временах, превышающих длительность либрационного отклика.

Данные предположения позволяют связать между собой величины $t_{\gamma}, \gamma^{(i)}$ и $\tau_{\mathrm{or}}^{(i)}$ при условии, что время нарастания ориентационного отклика $t_{\gamma}$ (соответствующего времени, когда ориентационный отклик $r_{\mathrm{or}}^{(i)}$ достигает своего максимума) принимается равным длительности либрационного отклика, $t_{\gamma}=\tau_{\mathrm{lib}}$ :

$$
\tau_{\mathrm{lib}}=\gamma^{(i)} \ln \left(\left(\gamma^{(i)}+\tau_{\mathrm{or}}^{(i)}\right) / \gamma^{(i)}\right) .
$$

Возвращаясь к рассмотренному выше примеру с сигналом ОЭК в бензонитриле, из рис. 1 можно оценить, что длительность либрационного отклика составляет величину $\tau_{\mathrm{lib}} \approx 0.3 \mathrm{ps}$. Таким образом, приведенные на рис. 2 варианты разложения оказываются несостоятельными, поскольку только в одном случае для параметров $\gamma^{(1)}=0.6 \mathrm{ps}, \tau_{\mathrm{or}}^{(1)}=0.4 \mathrm{ps}$ время нарастания ориентационного отклика оказалось близко к значению 0.3 ps. Принимая, что в ориентационном отклике (14) участвуют два релаксационных процесса с параметрами $\gamma^{(1)}=0.5 \mathrm{ps}, \tau_{\mathrm{or}}^{(1)}=0.4 \mathrm{ps}, \gamma^{(2)}=0.06 \mathrm{ps}$ и $\tau_{\mathrm{or}}^{(2)}=8 \mathrm{ps}$, удовлетворяющими (18) со значением $\tau_{\text {lib }}=0.3$ ps (рис. $\left.3, a\right)$, разделение вращательных откликов $r_{\mathrm{or}}(t)$ и $r_{\mathrm{lib}}(t)$ приводит к виду функции $T_{\mathrm{RSD}}(t)$, показанному на рис. $3, b$.

Следует отметить, что возможны и иные симуляции ориентационной функции $r_{\text {or }}(t)$ с двумя релаксационными процессами. Расчет показывает, что возможными

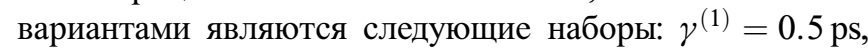
$\tau_{\mathrm{or}}^{(1)}=0.4 \mathrm{ps}, \quad \gamma^{(2)}=0.08 \mathrm{ps}, \quad \tau_{\mathrm{or}}^{(2)}=3 \mathrm{ps} ; \quad \gamma^{(1)}=0.3 \mathrm{ps}$, $\tau_{\mathrm{or}}^{(1)}=0.5 \mathrm{ps}, \gamma^{(2)}=0.07 \mathrm{ps}, \tau_{\mathrm{or}}^{(2)}=4 \mathrm{ps}$. В них параметры $\gamma^{(i)}$ и $\tau_{\text {or }}^{(i)}$ удовлетворяют уравнению (18) со значением $\tau_{\mathrm{lib}}=0.3 \mathrm{ps}$. Однако, несмотря на существенное различие в величинах, кривые ориентационных откликов очень близки к показанным на рис. 3,a вариантам, так что в диапазоне от 0 до 2 ps невозможно отдать предпочтение какому-либо одному. Естественно, что формы редуцированной спектральной плотности $T_{\mathrm{RSD}}(\omega)$, полученные для каждого варианта ориентационной функции $r_{\mathrm{or}}(t)$, близки виду функции $T_{\mathrm{RSD}}(\omega)$ на рис. $3, b$. Отсюда следует важный вывод, что при анализе экспериментального сигнала ОЭК в бензонитриле в диапазоне 0-2ps затруднительно определить времена релаксации ориентационной анизотропии $\tau_{\mathrm{or}}^{(i)}$. Известно, что для определения их точных значений необходимо существенно увеличить диапазон задержек до нескольких пикосекунд. Например, в работе [28] для бензонитрила приводят следующие значения времен релаксации ориентационного отклика: $\tau_{\mathrm{or}}^{(1)}=1.07 \mathrm{ps}$, $\tau_{\mathrm{or}}^{(2)}=4.96 \mathrm{ps}$. С учетом длительности либрационного отклика 0.3 ps этим временам будут соответствовать значения $\gamma^{(1)}=0.14 \mathrm{ps}$ и $\gamma^{(2)}=0.07 \mathrm{ps}$. Именно этот вариант, показанный на рис. 3 пунктирной линией, по 

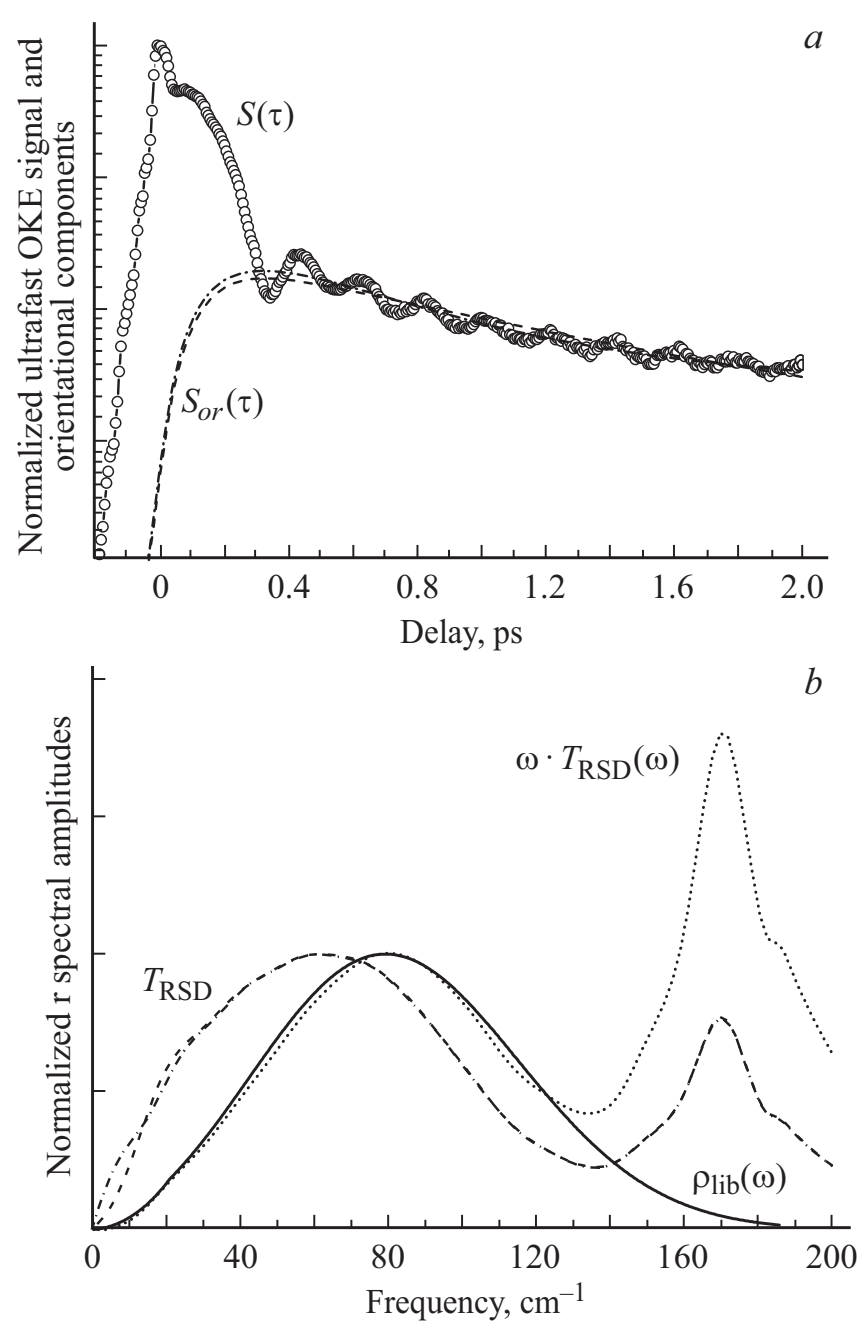

Рис. 3. (а) Сигнал сверхбыстрого ОЭК $S(\tau)$ в бензонитриле и ориентационные вклады $S_{\text {оr }}(\tau)$. $(b)$ Нормированные редуцированные рамановские спектральные плотности $T_{\mathrm{RSD}}(\omega)$, пунктир - функция $\omega T_{\mathrm{RSD}}(\omega)$, сплошная линия - либрационный спектр $\rho_{\text {lib }}(\omega)$ из [24]. Символами о обозначены экспериментальные данные; пунктиру соответствуют кривые, вычисленные с параметрами ориентационного отклика: $\tau_{\mathrm{or}}^{(1)}=1.07 \mathrm{ps}$, $\gamma^{(1)}=0.14 \mathrm{ps}, \tau_{\mathrm{or}}^{(2)}=4.96 \mathrm{ps}, \gamma^{(2)}=0.07 \mathrm{ps}$; штрихпунктиру кривые с параметрами ориентационного отклика: $\tau_{\mathrm{or}}^{(1)}=0.4 \mathrm{ps}$, $\gamma^{(1)}=0.5 \mathrm{ps}, \tau_{\text {or }}^{(2)}=8 \mathrm{ps}, \gamma^{(2)}=0.06 \mathrm{ps}$.

всей видимости, наиболее близок к истинному виду функции $T_{\mathrm{RSD}}(\omega)$.

Представляется важным провести сравнение с результатами работы [24], где разложение сигнала ОЭК было выполнено на основе сценария двухимпульсного возбуждения путем подавления ориентационного отклика. Наиболее корректно это сделать с помощью выражения [24]:

$$
\rho_{\mathrm{lib}}(\omega) \propto \omega T_{\mathrm{RSD}}(\omega)
$$

которое связывает в низкочастотной области спектр молекулярных либраций $\rho_{\mathrm{lib}}(\omega)$, полученный в [24], и вычисленную выше редуцированную спектральную плотность $T_{\mathrm{RSD}}(\omega)$. На рис. $3, b$ видно, что результаты хорошо согласуются в области $0-125 \mathrm{~cm}^{-1}$, которая как раз и обусловлена либрациями молекул. Различие в области частот, больших $125 \mathrm{~cm}^{-1}$, связано с присутствием в $T_{\mathrm{RSD}}(\omega)$ вклада комбинационно-активного внутримолекулярного колебания на частоте $170 \mathrm{~cm}^{-1}$. В [24] приведена также РСП, полученная на основе традиционного анализа экспериментальных данных с одноимпульсным возбуждением ОЭК, где в качестве ориентационного отклика было использовано выражение (1) без учета корреляции между либрационным и ориентационным откликами. Важно отметить, что эти данные отличны от функции РСП на рис. $3, b$, поскольку они дают существенное расхождение со спектром молекулярных либраций $\rho_{\mathrm{lib}}(\omega)$ в области частот, больших $100 \mathrm{~cm}^{-1}$.

\section{Заключение}

В работе представлен детальный анализ низкочастотных молекулярных откликов в терагерцовой области, которые регистрируется с высоким соотношением „сигнал/шум“ в сигнале сверхбыстрого ОЭК, представляющего собой оптический нелинейный отклик среды третьего порядка при нерезонансном возбуждении жидкостей лазерными фемтосекундными импульсами. Основной акцент сделан на проблеме разделения ориентационного и либрационного вкладов в совокупный сигнал ОЭК с помощью хорошо известной процедуры деконволюции [11-13]. На примере анализа сигнала ОЭК в бензонитриле показано, что в случае применения традиционного подхода $[5,6,12,13]$, в котором вид ориентационного отклика описывается выражением (1), наблюдается зависимость формы редуцированной рамановской спектральной плотности от параметра $\gamma$, описывающего нарастание ориентационного отклика. На основании качественного анализа механизмов вращательной диффузии, участвующих в релаксации ориентационной анизотропии молекул в жидкости, сделан вывод о том, что каждому релаксационному процессу со временем $\tau_{\text {or }}^{(i)}$ должно соответствовать время нарастания $\gamma^{(i)}$. В результате чего предложено выражение (14) для более точного симулирования особенностей ориентационного отклика.

Традиционно считается, что все отклики, формирующие сигнал ОЭК, являются независимыми друг от друга. В настоящей работе указывается на необходимость учитывать корреляцию между либрационным и ориентационным откликами, поскольку в их основе лежат молекулярные вращения. Отметим, что выяснение особенностей этой корреляции требует дальнейших интенсивных экспериментальных и теоретических исследований. Однако качественные предположения о характере корреляции позволяют связать между собой длительность либрационного отклика $\tau_{\mathrm{lib}}$, времена нарастания $\gamma^{(i)}$ и релаксации ориентационного отклика $\tau_{\text {or }}^{(i)}$. Учет корреляции с помощью выражения (18) в значительной 
мере снимает проблему неоднозначного разделения вращательных откликов при получении функции РСП из сигнала ОЭК с помощью процедуры деконволюции.

При анализе низкочастотных движений молекул в бензонитриле были приняты во внимание следующие факты. (i) Значения времен релаксации $\tau_{\mathrm{or}}^{(i)}$ определены c помощью экспериментальных данных о поведении ориентационного отклика в пикосекундном диапазоне. (ii) Длительность либрационного отклика $\tau_{\text {lib }}$ была оценена по виду сигнала ОЭК в области сотен фемтосекунд. (iii) Из выражения (18) с учетом данных о длительности либрационного отклика и временах релаксации ориентационного отклика получены значения времен нарастания ориентационного отклика $\gamma^{(i)}$ для его симуляции выражением (14). (iv) Найденный вид ориентационного отклика позволил вычесть из наблюдаемого сигнала ОЭК ориентационный вклад (7), после чего с помощью процедуры деконволюции (6) получен искомый вид функции РСП, отражающей особенности низкочастотной молекулярной динамики и локальной организации жидкости в фемтосекундном диапазоне.

\section{Список литературы}

[1] Nielsen O.F. // Ann. Rep. Prog. Chem. C. 1997. V. 93. P. 57.

[2] Kinoshita S., Kai Y., Ariyoshi T., Shimada Y. // Int. J. Mod. Phys. B. 1996. V. 10. P. 1229.

[3] Castner E.W., Wishart J.F., Shirota H. // Acc. Chem. Res. 2007. V. 40. P. 1217.

[4] Shirota H., Kato T. // J. Phys. Chem. A. 2011. V. 115. P. 8797.

[5] Zhong Q., Fourkas J.T. // J. Phys. Chem. B. 2008. V. 112. P. 15529.

[6] Hunt N.T., Jaye A.A., Meech S.R. // Phys. Chem. Chem. Phys. 2007. V. 9. P. 2167.

[7] Kinoshita S., Kai Y., Yamaguchi M., Yagi T. // Chem. Phys. Lett. 1995. V. 236. P. 259.

[8] Nakayama H., Yajima S., Yoshida T., Ishii K. // J. Raman Spectrosc. 1997. V. 28. P. 15.

[9] Beard M.C., Turner G.M., Schmuttenmaer C.A. // J. Phys. Chem. B. 2002. V. 106. P. 7146.

[10] Самарцев В.В., Никифоров В.Г. Фемтосекундная лазерная спектроскопия. М.: Тровант, 2017. 402 с.

[11] McMorrow D. // Opt. Commun. 1991. V. 86. P. 236.

[12] McMorrow D., Lotshaw W.T. // Chem. Phys. Lett. 1990. V. 174. P. 85

[13] McMorrow D., Lotshaw W.T. // J. Phys. Chem. 1991. V. 95. P. 10395.

[14] Ryu S., Stratt R.M. // J. Phys. Chem. B. 2004. V. 108. P. 6782.

[15] Tao G., Stratt R.M. // J. Phys. Chem. B. 2006. V. 110. P. 976.

[16] Elola M.D., Ladanyi B.M. // J. Phys. Chem. B. 2006. V. 110. P. 15525.

[17] Yang P., Voth G.A., Xiao D., Hines L.G. Jr., Bartsch R.A., Quitevis E.L. // J. Chem. Phys. 2011. V. 135. P. 034502.

[18] Lynden-Bell R.M., Xue L., Tamas G., Quitevis E.L. // J. Chem. Phys. 2014. V. 141. P. 0044506.

[19] Bender J.S., Fourkas J.T., Coasne B. // J. Phys. Chem. B. 2017. V. 121. P. 11376

[20] Zhu X., Farrer R.A., Fourkas J.T. // J. Phys. Chem. B. 2005. V. 109. P. 8481.
[21] Nikiforov V.G., Shmelev A.G., Safiullin G.M., Lobkov V.S. // Appl. Phys. Lett. 2012. V. 100. P. 081904-1.

[22] Nikiforov V.G. // Quant. Electron. 2013. V. 43. P. 177.

[23] Nikiforov V.G., Shmelev A.G., Safiullin G.M., Lobkov V.S. // Chem. Phys. Lett. 2014. V. 592. P. 196.

[24] Nikiforov V.G., Zharkov D.K., Shmelev A.G., Leontyev A.V., Lobkov V.S. // Appl. Phys. B. 2017. V. 123. P. 209.

[25] Smith N.A., Meech S.R. // J. Phys. Chem. A. 2000. V. 104. P. 4223.

[26] Chang Y.J., Cong P., Simon J. // J. Chem. Phys. 1997. V. 106. P. 8639.

[27] Cong P., Deuel H.P., Simon J.D. // Chem. Phys. Lett. 1995. V. 240. P. 72.

[28] Shirota H., Fujisawa T., Fukazawa H., Nishikawa K. // Bull. Chem. Soc. Jpn. 2009. V. 82, P. 1347.

[29] Френкель Я.И. Кинетическая теория жидкости. М.: Издательство Академии Наук СССР. 1945. 424 с.

[30] McConnell J. Rotational brownian motion and dielectric theory. London: Academic Press, 1980. 422 p.

[31] Debye P. Polar Molecules. N.Y.: Dover Publications, Inc. 1928. $172 \mathrm{p}$.

[32] Burshtein A.I., Temkin S.I. Spectroscopy of Molecular Rotation in Gases and Liquids. N.Y.: Cambridge University Press, 2005. 300 p.

[33] Wang Y., Ushida K., Tominaga Y., Kira A. // Chem. Phys. Lett. 1999. V. 299. P. 576. 